\title{
CIÊNCIA'NATURA
}

\section{VARIACÕES DE INTENSIDADE DO CAMPO GEOMAGNÉTICO EM SANTA MARIA (BRASIL) PARA OS ULTIMOS 3 MIL ANOS}

\author{
Intensity variations of geomagnetic field in Santa Maria (Brazil) over the past 3 thousand \\ years
}

\author{
Everton Frigo ${ }^{1}$ e Gelvam A. Hartmann ${ }^{2}$
}

'Universidade Federal do Pampa, Campus Caçapava do Sul, Caçapava do Sul, Brasil

${ }^{2}$ Instituto de Geociências, Universidade Estadual de Campinas, Campinas, Brasil

\begin{abstract}
Resumo
As variações do campo magnético terrestre em escala temporal secular e escala espacial da ordem de centenas de quilômetros geralmente tem origem no núcleo externo da Terra. A feição mais marcante associada ao campo geomagnético interno é a Anomalia Magnética do Atlântico Sul (AMAS), que atualmente cobre todo o sul da América do Sul, e é caracterizada pelas menores intensidades do campo geomagnético em toda a superficie da Terra. Neste trabalho investigamos as variações geomagnéticas temporais para os últimos 3 mil anos, registradas em Santa Maria, localizada na área influenciada pela AMAS. Os resultados indicam que as intensidades geomagnéticas observadas atualmente são as mais baixas dos últimos 3 mil anos. Como consequência destes fenômenos, o aumento na incidência de partículas eletricamente carregadas que chegam até a baixa atmosfera da Terra, pode gerar problemas desde sistemas de comunicação até mesmo no clima do planeta.
\end{abstract}

Palavras-chave: Campo Geomagnético. Anomalia Magnética do Atlântico Sul. Santa Maria

\section{Abstract}

Earth magnetic field variations at secular scales and at hundred quilometers have internal origin at the outer core. The most important feature associated with this internal field is the South Atlantic Magnetic Anomaly (SAMA), which covers the South America and it is characterized by the lowest total field intensity at the surface. Here, we investigate the geomagnetic field variations in Santa Maria (Brazil) over the past $3 \mathrm{ka}$. Results indicate that the intensities observed in Santa Maria are the lowest of the geomagnetic field over the past $3 \mathrm{ka}$. The consequences of these field features could be the increase in the electrically charged particles reaching the low Earth atmosphere that may generate problems in the communications systems or climate changes.

Keywords: Geomagnetic field. South Atlantic Magnetic Anomaly. Santa Maria 


\section{Introdução}

O campo magnético da Terra (CMT) é gerado no núcleo externo através da movimentação do fluido eletricamente condutor. Esses movimentos convertem a energia mecânica em energia eletromagnética, produzindo assim um dínamo auto-sustentado, o chamado geodínamo (e.g. Hulot et al., 2010). Os movimentos das correntes elétricas induzem um campo magnético, que tem forma predominantemente dipolar devido a rotação da Terra. Esse campo dipolar apresenta, na média, aproximadamente $65.000 \mathrm{nT}$ nas regiões polares e em torno de $30.000 \mathrm{nT}$ na região equatorial. O campo geomagnético se estende no espaço ao redor da Terra, formando uma região denominada magnetosfera, na qual o campo terrestre controla os fenômenos eletromagnéticos que ali ocorrem. No lado dia da Terra, a magnetosfera se estende por uma distância de até dez raios terrestres a partir do centro da Terra, enquanto que no lado noite a magnetosfera pode atingir até centenas de raios terrestres (CAMPBELL, 2003). O CMT funciona como um escudo protetor que protege nosso planeta da entrada de partículas eletricamente carregadas provenientes do espaço. Essas partículas, por sua vez, podem produzir efeitos indesejáveis em sistemas eletromagnéticos de aeronaves, causar danos à saúde humana, prejudicar os sistemas de transmissão de energia elétrica e até mesmo afetar o clima (e.g. Hulot et al., 2010).

Desde as primeiras medidas diretas de intensidade total, de declinação e de inclinação geomagnética, foi possível concluir que o CMT apresenta variações espaciais e temporais. Esta variabilidade foi denominada variação geomagnética secular. A instalação de observatórios para a realização de monitoramento contínuo do CMT possibilitou o entendimento da variação geomagnética diurna, que em períodos de baixa atividade solar é caracterizada por uma curva de mínima intensidade total no início do dia, passando por um máximo próximo ao meio dia e voltando ao mínimo durante a noite. O avanço nas técnicas e equipamentos de medidas de magnetização tornaram possíveis também medidas geomagnéticas indiretas, realizadas em amostras de rochas e artefatos arqueológicos. Estas medidas, permitiram obter valores de declinação e inclinação geomagnética correspondente ao campo geomagnético presente na época em que esses materiais se formaram. As informações advindas dessas medidas evidenciaram a existência das excursões geomagnéticas e das reversões de polaridade (MERRILL et al., 1998).

A reunião de conjuntos de dados geomagnéticos permitiu a construção de modelos de campo. Estes modelos se diferenciam entre si pelos conjuntos de dados utilizados e pelas premissas básicas de cada um. Atualmente existem modelos geomagnéticos que permitem descrever o campo para os últimos três mil anos. Esses modelos evidenciam importantes feições regionais do CMT na superfície da Terra e permitem investigar suas possíveis origens na interface manto-núcleo. Uma das maiores e mais investigadas feições do CMT é a Anomalia Magnética do Atlântico Sul (AMAS), cujo centro se deslocou do sul da África para o sul da América do Sul nos últimos quatro séculos (e.g Hartmann e Pacca, 2009; Terra-Nova et al., 2017). Além deste deslocamento para o oeste, a AMAS tem aumentado sua área de influência desde $1590 \mathrm{AD}$. Com a aproximação e passagem do centro da AMAS pelo sul do Brasil, a intensidade do campo geomagnético nesta região vem apresentando uma forte tendência de decréscimo.

O objetivo deste trabalho é utilizar dados de modelos de campo geomagnético para descrever as variações geomagnéticas de intensidade em Santa Maria, para os últimos três mil anos, e discutir as mesmas em termos da fenomenologia do campo geomagnético e de suas possíveis consequências para as atividades humanas.

\section{Modelos de Campo Geomagnético}

Os dados geomagnéticos são fundamentais para o entendimento de aspectos relacionados ao mecanismo de geração e as variações espaciais e temporais do CMT. No entanto, em razão de vários fatores como as dimensões envolvidas, dificuldade de acesso e o alto custo, não é possível realizar o monitoramento contínuo do campo geomagnético em todas as posições da superfície terrestre. Este problema é, em parte, resolvido através da utilização de modelos de campo geomagnético, que consistem basicamente de equações físico-matemáticas que são ajustadas por dados geomagnéticos medidos em diferentes locais da Terra ou de regiões próximas. Os modelos geomagnéticos mais utilizados atualmente são baseados na representação do campo geomagnético por harmônicos esféricos, que foi desenvolvida por Gauss nas primeiras décadas do século XIX. A equação básica desta representação descreve o potencial escalar associado ao vetor campo geomagnético para cada posição, em função da latitude e da longitude, e da distância em relação ao centro da Terra. A utilização de medidas geomagnéticas, tomadas em algumas localidades, permite calcular os coeficientes dos harmônicos esféricos e, a partir destes é possível predizer os valores de campo geomagnético para locais para os quais não existem medidas de campo. Os modelos mais utilizados para descrever o campo geomagnético nos últimos três mil anos são o International Geomagnetic Reference Field (IGRF, Thébault et al., 2015), o gufm 1 (Jackson et al., 2000) e o Continuous Archeomagnetic and Lake Sediment Geomagnetic Model (CALS3k.4, Korte \& Constable, 2011).

O IGRF é mantido e atualizado a cada cinco anos pela International Association on Geomangetism and Aeronomy (IAGA), sendo a última atualização realizada em 2015 (THEBAULT et al., 2015). Este modelo é construído a partir do ajuste do modelo de harmônicos esféricos a medidas geomagnéticas realizadas em observatórios geomagnéticos, estações geomagnéticas fixas e móveis e satélites. É o modelo de campo geomagnético mais preciso para o período $1900 \mathrm{AD}-2015 \mathrm{AD}$ e amplamente 
utilizado em estudos do campo geomagnético de origem interna.

Para o período 1590 AD - 1990 AD, o modelo geomagnético mais utilizado é o gufm1 (Jackson et al., 2000). Este modelo se utiliza de dados direcionais de declinação e inclinação geomagnética, baseados nos registros dos navegadores da época das Grandes Navegações. Em razão da escassez de dados de intensidade, anteriores às primeiras medidas de Gauss em 1840, o gufm1 utilizou o valor constante de decréscimo do campo dipolar de $15 \mathrm{nT} / \mathrm{ano}$, valor este que equivale a média global observada entre 1840 e 1990.

O CALS3k.4 (Korte \& Constable, 2011) é o modelo mais preciso para os últimos 3 mil anos. Este modelo utiliza informações do IGRF para o período $1900 \mathrm{AD}$ - $1990 \mathrm{AD}$, do modelo gufm1 para o período $1590 \mathrm{AD}-1990 \mathrm{AD}$ e, une a estes dados arqueomagnéticos e de sedimentos recentes de lagos do período $1000 \mathrm{BC}-1900 \mathrm{AD}$. Os dados de intensidade obtidos a partir de materiais arqueológicos são determinados a partir de medidas realizadas em magnetômetros extremamente sensíveis e seguindo um rigoroso procedimento experimental (e.g. HARTMANN, 2010). Os dados obtidos em laboratório são registrados em artefatos arqueológicos, principalmente cerâmicas, telhas e tijolos, a partir do aquecimento de argila em temperaturas acima da temperatura de Curie (ou de Néel) dos minerais ferrimagnéticos portadores de magnetização. Quando esses minerais são resfriados a partir da temperatura de Curie, na presença do campo geomagnético, eles adquirem uma magnetização com intensidade proporcional à intensidade do CMT à época em que esses materiais foram produzidos.

\section{Variações da intensidade total do campo geomagnético em Santa Maria}

A cidade de Santa Maria está localizada na região central do estado do Rio Grande do Sul, sul do Brasil (Figura 1). Esta região vem sendo fortemente influenciada pela AMAS desde o início do século XX. Atualmente, os valores de intensidade geomagnética na região de Santa Maria são inferiores a $22.500 \mathrm{nT}$ (ou 22,5 $\mu \mathrm{T}$ ). Note que esses valores são inferiores até mesmo àqueles observados na região equatorial.

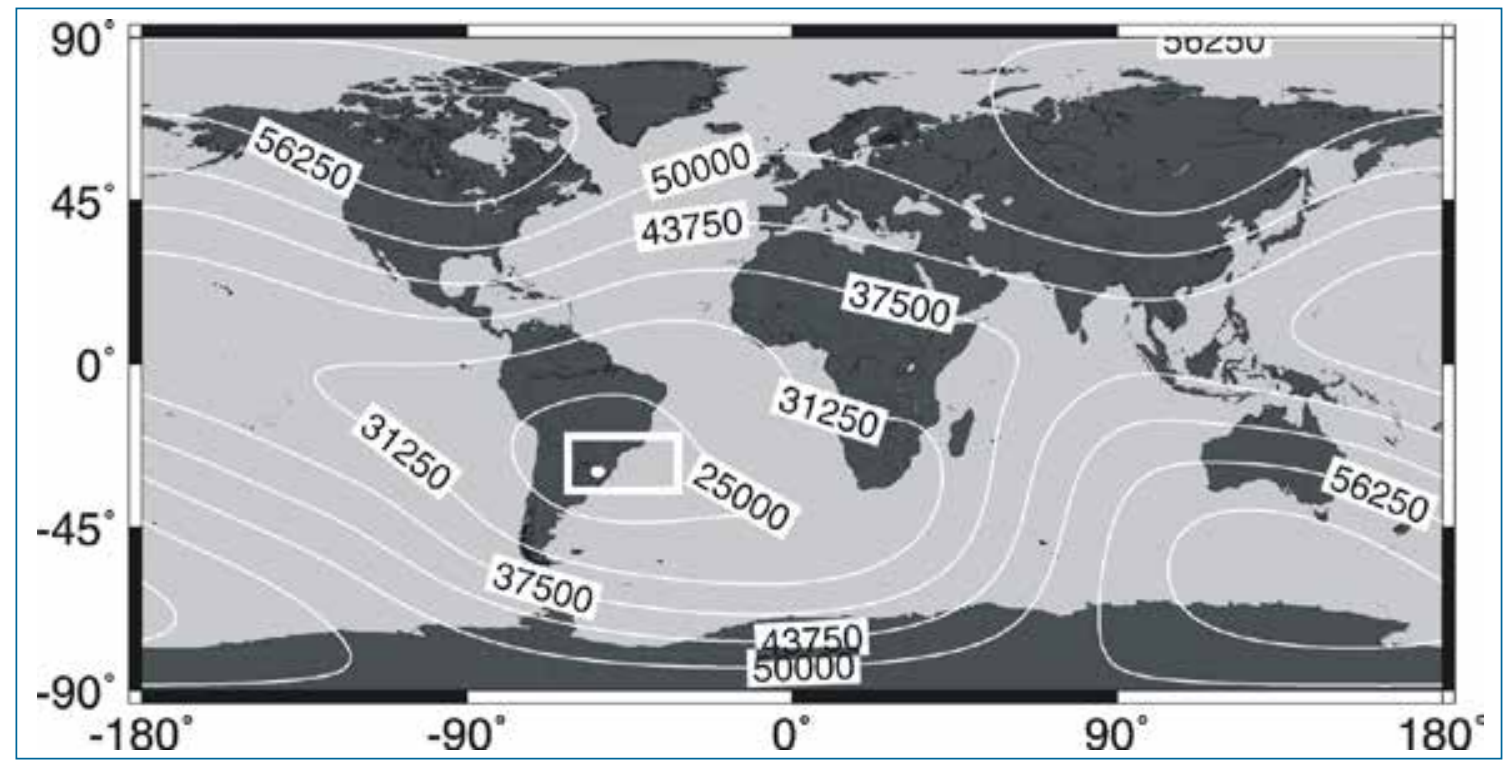

Figura 1 - Mapa global de intensidade total do campo geomagnético, para o ano 2010, obtido a partir do modelo IGRF. Parte da região sul da América do Sul, indicada pela área retangular na cor branca, está inserida na região de menor intensidade geomagnética observada na superfície terrestre. A cidade de Santa Maria está indicada pelo círculo branco.

A Figura 2 mostra a variação da intensidade do campo geomagnético para os últimos 3 mil anos para calculada para as coordenadas da cidade de Santa Maria. Essas variações foram calculadas pelos modelos CALS3k.4, para o período 1000 BC - 1990 AD, e IGRF 2015, para o período 1900 AD - 2015 AD. Apesar de se observar épocas em que o campo geomagnético aumentou em intensidade durante décadas a séculos, é notável a tendência de decréscimo da intensidade do CMT nos últimos 3 mil anos para Santa Maria. Mais acentuada ainda é a forte queda de intensidade verificada entre 1650 e 2015.

Entre $1000 \mathrm{BC}$ e pouco depois de $250 \mathrm{BC}$, a intensidade do CMT apresentou uma tendência de decréscimo de pouco mais de $50 \mu \mathrm{T}$ até por volta de $44 \mu \mathrm{T}$. Durante estes 750 anos são observadas duas épocas, entre $620 \mathrm{BC}$ e $540 \mathrm{BC}$ e entre 445 $\mathrm{BC}$ e $315 \mathrm{BC}$, em que a intensidade do campo geomagnético aumentou de poucos nanoteslas. Após $215 \mathrm{BC}$ verifica-se um 
período de em que a intensidade do CMT aumentou de 43,7 $\mu \mathrm{T}$ para $51 \mu \mathrm{T}$ em $20 \mathrm{BC}$. Estes quase dois séculos de aumento na intensidade geomagnética seguiram-se por uma forte diminuição, que durou aproximadamente 500 anos e fez o campo decrescer de $\sim 50 \mu \mathrm{T}$ para $\sim 35 \mu \mathrm{T}$. Entre $500 \mathrm{AD}$ e $885 \mathrm{AD}$ a intensidade do campo permaneceu relativamente estável, com valores oscilando ao redor de $\sim 37 \mu \mathrm{T}$.

A partir de $885 \mathrm{AD}$ a intensidade geomagnética aumentou significativamente durante um período de $\sim 255$ anos, atingindo o valor máximo para os últimos 3 mil anos, de 53,3 $\mu \mathrm{T}$, em 1140 AD. Durante os 150 anos subsequentes, o campo apresentou uma tendência de decréscimo atingindo o valor de 43,6 $\mu \mathrm{T}$ em 1290 AD. Após, a intensidade aumentou até atingir 47,4 $\mu \mathrm{T}$ em 1390 AD. Depois, até $1500 \mathrm{AD}$, ocorreu um decréscimo na intensidade do campo geomagnético, chegando ao valor de $45 \mu \mathrm{T}$. Posteriormente, o campo passou por outra época de aumento de intensidade, sendo verificado o valor de 49,2 $\mu \mathrm{T}$ em $1645 \mathrm{AD}$.

As variações de intensidade geomagnética observadas desde 1000 BC até 1645 AD são entendidas como manifestações típicas da variação geomagnética secular. A variação secular é caracterizada por mudanças de alguns nanotesla por ano na intensidade geomagnética e por deslocamentos dos polos magnéticos, ao redor dos polos geográficos, não superiores a $10^{\circ}$ de latitude. Estas variações, ocorrem em consequência de mudanças lentas no interior terrestre, principalmente no núcleo externo terrestre, onde o campo geomagnético é gerado (MERRILL et al., 1998; HULOT et al., 2010).

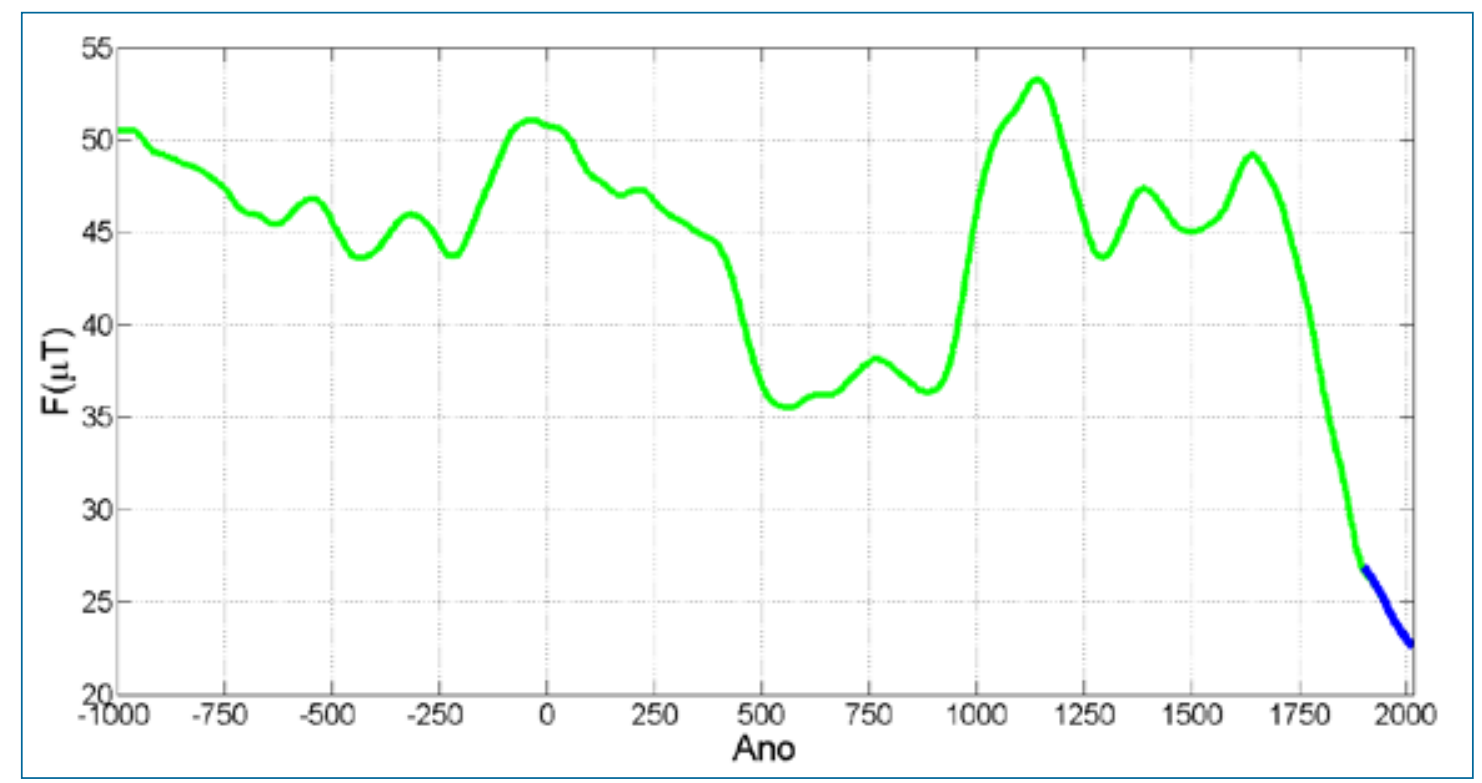

Figura 2 - Intensidade do campo geomagnético obtida a partir dos modelos CALS3k.4 (curva verde) e do IGRF 2015 (curva azul). Para o período 1900 AD - 1990 AD ocorre uma sobreposição entre as duas curvas.

Após $1645 \mathrm{AD}$, é observado o maior decréscimo de intensidade geomagnética para os últimos 3 mil anos. Nestes últimos 370 anos de queda, a intensidade do campo atual, de $\sim 22,5 \mu \mathrm{T}$, equivale a aproximadamente $45 \%$ do valor máximo observado nos últimos 3 mil anos ou aproximadamente $46 \%$ do valor observado em 1645 AD. Este decréscimo pode ser explicado pela aproximação da AMAS, cujo centro se deslocou do sul do continente africano para o sul da América do Sul nos últimos 400 anos. A origem e o deslocamento da AMAS podem estar relacionados com a topografia da interface manto-núcleo externo da Terra (HARTMANN \& PACCA, 2009; TERRA-NOVA et al., 2016).

Por outro lado, este forte decréscimo de intensidade observado nos últimos séculos pode estar associado a uma excursão geomagnética, fenômeno em que a intensidade geomagnética cai significativamente acompanhada de um afastamento do polo magnético em relação ao polo geográfico, de menos de 45 graus de latitude, com posterior retorno ao ciclo de variação secular nas proximidades do polo geográfico. Tipicamente, este fenômeno dura poucos séculos. Outra possibilidade, ainda mais drástica, seria o início de uma inversão de polaridade geomagnética, que também é caracterizada pela forte queda de intensidade do CMT, até valores inferiores a 20\% dos valores antes do início do decréscimo. Quando ocorre uma inversão de polaridade, o polo magnético que realizava o ciclo de variação secular ao redor do polo geográfico norte, por exemplo, passa a realizar o ciclo ao redor do polo geográfico sul e, permanece nesta situação até que uma nova inversão ocorra. O tempo necessário para que o fenômeno de inversão se complete é de poucos milhares de anos. A última inversão registrada ocorreu a aproximadamente 780 mil de anos e, a taxa de inversões varia entre 0 e 4 reversões a cada 1 milhão de anos. Atualmente, a frequência de inversões é a mais alta desde os últimos 160 milhões de anos (GUBBINS, 1999; HULOT et al., 2010).

As principais consequências do enfraquecimento do campo geomagnético nos últimos séculos para a vida humana estão relacionadas com o aumento de partículas eletricamente carregadas na atmosfera terrestre (PINTO JR et al., 1992). Estas par- 
tículas podem induzir correntes elétricas nos sistemas eletrônicos de satélites ou mesmo aviões, danificando estes sistemas e prejudicando o funcionamento dos mesmos. Se a intensidade geomagnética continuar diminuindo, num futuro não tão distante poderão ocorrer situações extremas de interrupções parciais, ou até mesmo totais, de sistemas de telecomunicações, sistemas de posicionamento global e até mesmo graves acidentes envolvendo aeronaves comerciais. Além disso, o campo geomagnético pouco intenso aliado a períodos de alta atividade solar, pode estar relacionado a maior incidência de radiações nocivas ao corpo humano, situação que não foi observada em períodos de atividade solar baixa (BAHDWAR et al., 2002; FEDERICO, 2011; FEDERICO et al., 2015).

Outro fenômeno, relacionado ao CMT, que pode influenciar a vida na Terra são as correntes elétricas induzidas geomagneticamente (GICs). As GICs ocorrem geralmente em dias de atividade solar acima da média, nos quais uma grande quantidade de partículas eletricamente carregadas penetra a barreira imposta pelo campo geomagnético e causam variações nas correntes elétricas que fluem na região da ionosfera terrestre. Estas correntes ionosféricas, por sua vez, induzem correntes elétricas em objetos metálicos de grande extensão como, por exemplo, as linhas de transmissão de energia elétrica. Nas linhas de transmissão, estas correntes elétricas de origem geomagnética podem causar severos danos em transformadores e levar ao desabastecimento de energia elétrica na região afetada (PIRJOLA, 2002). As GICs ocorrem geralmente em regiões de altas latitudes, onde o campo geomagnético é predominantemente vertical oferecendo pouca resistência a entrada de partículas carregadas. No entanto, se a intensidade geomagnética continuar diminuindo de intensidade, este tipo de fenômeno também poderá vir a afetar a região influenciada pela AMAS. Alguns estudos realizados no Brasil (TRIVEDI et al., 2007; BARBOSA et al., 2015a, 2015b) e Uruguai (CARABALLO et al., 2013) já detectaram eventos de GICs na América do Sul.

Nos últimos anos, o campo geomagnético também vem sendo sugerido como um forçante natural, que atua de forma indireta, sobre o clima terrestre. Esta atuação se dá através da modulação que o CMT exerce sobre os raios cósmicos galácticos (RCGs), que são partículas geralmente com carga elétrica positiva geradas a partir de supernovas, e cuja ionização é uma importante fonte de energia para a baixa atmosfera terrestre. Segundo este mecanismo, a ionização produzida pelos RCGs contribui para a formação de aerossóis, que podem crescer e formar núcleos de condensação de nuvens, que são necessários para a condensação de gotas de água ao seu redor e consequente formação de nuvens de baixa altitude (SVENSMARK \& FRISS-CHRISTENSEN, 1997; SVENSMARK, 2015. Assim, uma menor intensidade total do CMT está relacionada a um maior fluxo de RCG e consequentemente a uma maior quantidade de nuvens baixas que, por sua vez, tem efeito de diminuir a temperatura abaixo de sua região de incidência. Esta possível influência sobre a temperatura pode ter também efeitos sobre outras variáveis meteorológicas como pressão atmosférica e precipitação pluviométrica. Trabalhos publicados recentemente por Rampelotto et al. (2012) e Frigo et al. (2013) apresentaram indicativos estatísticos de que a presença da AMAS pode ter influenciado a variabilidade de precipitação pluviométrica e temperatura na região sul do Brasil durante o último século.

\section{Conclusões}

O campo geomagnético de origem interna apresenta variações temporais e espaciais. Estas variações são conhecidas a partir de medidas geomagnéticas diretas e indiretas. A partir do agrupamento de diferentes tipos de dados geomagnéticos é possível construir modelos de campo que reproduzem a evolução temporal e espacial da intensidade geomagnética para os últimos milênios.

A intensidade total do CMT para Santa Maria para os últimos três mil anos oscilou entre 40 e $50 \mu$ T. Valores um pouco mais baixos, entre 35 e $40 \mu \mathrm{T}$ foram observados entre $\sim 500 \mathrm{AD}$ e $\sim 950 \mathrm{AD}$. As variações de intensidade do campo entre 1000 $\mathrm{BC}$ e $1645 \mathrm{AD}$ pode ser consideradas manifestações típicas da variação geomagnética secular. No entanto, a mudança mais marcante na intensidade do campo geomagnético é observada após $1645 \mathrm{AD}$, no qual a intensidade começou a seguir uma forte tendência de decréscimo, partindo de 49,2 $\mu \mathrm{T}$ e atingindo o valor de $\sim 22,5 \mu \mathrm{T}$ em 2015 . Esta forte queda na intensidade do campo pode ser atribuída a aproximação da AMAS e passagem do seu centro pelo sul do Brasil.

Este enfraquecimento da barreira geomagnética, verificado nos últimos quatro séculos, pode levar a um aumento no índice de falhas em sistemas eletrônicos embarcados em veículos aéreos. Além disso, pode ocorrer um aumento na quantidade de eventos de correntes elétricas geomagneticamente induzidas em regiões de baixas latitudes. A queda na intensidade do campo pode contribuir também para o aumento de efeitos danosos a saúde humana devido a radiação, principalmente me períodos de alta atividade solar. Por fim, alguns estudos têm apresentado indícios de que a baixa intensidade geomagnética na região influenciada pela AMAS pode estar afetando, indiretamente, o clima da região sul do Brasil.

\section{Agradecimentos}

Agradecimentos a revisores, colaboradores e agências de fomento. 


\section{Referências}

Badhwar GD, Atwell W, Reitz G, Beaujean R, Heinrich H. Radiation measurements on the Mir Orbital Station. Radiat. Meas. 2002; 35 (2002):393-422.

BARBOSA CS, HARTMANN GA, PINHEIRO KJ. Numerical modeling of geomagnetically induced currents in a Brazilian transmission line. Adv. Space Res. 2015; 55:1168-1179.

BARBOSA Cs,ALVES L, CARABALLO R, Hartmann GA, PAPA ARR, PIRJOLA RJ. Analysis of geomagnetically induced currents at a low-latitude region over the solar cycles 23 and 24: comparison between measurements and calculations. Journal of Space Weather and Space Climate. 2015; 5: A35.

CAMPBELL WH. Introduction to Geomagnetic Fields. 2nd ed. Cambridge: University Press; 2003.

Caraballo R, Sanchez Bettucci L, Tancredi G. Geomagnetically induced currents in the Uruguayan high-voltage power grid. Geophys. J. Int. 2013; 195:844-853.

FEDERICO CA. Dosimetria da radiação cósmica no interior de aeronaves no espaço aéreo brasileiro [thesis]. São Paulo: Instituto de Pesquisas Energéticas e Nucleares/USP; 2011. 172 p.

Federico CA, Gonçalez OL, Caldas LVE, Pazianotto MT, Dyer C, Caresana M, Hands A. Radiation Measurements Onboard Aircraft in the South Atlantic Region Radiat. Meas. 2015; doi: 10.1016/j.radmeas.2015.07.008

Frigo E, Pacca IG, Pereira-Filho AJ, Rampelloto, PH, Rigozo, NR. Evidence for cosmic ray modulation in temperature records from the South Atlantic Magnetic Anomaly region. Ann. Geophys. 2013; 31:1833-1841.

GUBBINS D. The distinction between geomagnetic excursions and reversals. Geophys. J. Int. 1999; 137: F1-F3.

HARTMANN GA. A Anomalia Magnética do Atlântico Sul: Causas e Efeitos [dissertation]. São Paulo: Instituto de Astronomia, Geofísica e Ciências Atmosféricas/USP; 2005. 151 p.

HARTMANN GA, PACCA IG. Time evolution of the South Atlantic Magnetic Anomaly. An. Acad. Bras. Ciênc. 2009; 81(2):243-255.

Hulot G, Finlay cc, Constable cg, Olsen n, Mandea m. The Magnetic Field of Planet Earth. Space Sci. Rev. 2010; 152:159-222.

Jackson A, Jonkers ART, Walker M. Four centuries of geomagnetic secular variation from historical records. Phil. Trans. R. Soc. London A. 2000; 358: 957-990.

Korte M, Constable C. Improving geomagnetic field reconstructions for 0-3 ka. Phys. Earth Planet. Inter. 2011; 188: 247-259.

Merrill RT, McElhinny MW, McFadden PL. The magnetic field of the Earth: Paleomagnetism, the core, and the deep mantle. 1st ed. Amsterdam: Elsevier Academic Press; 1998.

Pinto Jr O, Gonzalez WD, Pinto IR, Gonzalez AC, Mendes Jr O. The South Atlantic Magnetic Anomaly: three decades of research. J Atmos Solar Terrest Phys. 1992; 54: 1129-1134.

PIRJOLA R. Review On The Calculation Of Surface Electric And Magnetic Fields And Of Geomagnetically Induced Currents In Ground-Based Technological Systems. Surv. Geophys. 2002; 23:71-90.

Rampelotto PH, Rigozo NR, da Rosa MB, Prestes A, Frigo E, Souza Echer MP, Nordemann DJR. Variability of rainfall and temperature (1912-2008) parameters measured from Santa Maria $\left(29^{\circ} 41^{\prime} S, 53^{\circ} 48^{\prime} \mathrm{W}\right)$ and their connections with ENSO and solar activity. J. Atmos. Solar-Terr. Phys. 2012; 77:152-160. 
SVENSMARK H. Cosmic rays, clouds and climate. Europhysics News. 2015; doi: 10.1051/epn/2015204.

Svensmark H, Friis-Christensen E. Variation of Cosmic Ray Flux and Global Cloud Coverage - a Missing Link in Solar-Climate relationships. J. Atmos. Solar-Terr. Phys. 1997; 59: 1225-1232.

TERRA-NOVA F, AMIT H, HARTMANN GA, TRINDADE RIF, PINHEIRO KJ. Relating the South Atlantic Anomaly and geomagnetic flux patches. Phys. Earth Planet. Int. 2017; 266:39-53.

THÉB AULT E, FINLAY CC, BEGGAN CD, ALKEN P, AUBERT J, BARROIS O, BERTRAND F, BONDAR T, BONESS A, BROCCO L, CANET E et al. Earth Plan. Space. 2015; 67-79.

TRIVEDI NB, VITORELLO I, KABATA W, DUTRA SLG, PADILHA AL, BOLOGNA MS, PADUA MB, SOARES AP, LUZ GS, PINTO FA, PIRJOLA R, VILJANEN A. Geomagnetically induced currents in an electric power transmission system at low latitudes in Brazil: A case study, Space Weather. 2007; 5: S04004. doi:10.1029/2006SW000282.

\section{Everton Frigo}

Universidade Federal do Pampa, Campus Caçapava do Sul, Caçapava do Sul, Brasil Email: evertonfrigo@unipampa.edu.br

Participação do autor: estruturação do artigo, da preparação das figuras, da discussão dos resultados e da redação do texto.

Gelvam A. Hartmann

Instituto de Geociências, Universidade Estadual de Campinas, Campinas, Brasil

Email: gelvam@ige.unicamp.br

Parcipação do autor: estruturação do artigo, da discussão dos resultados e da redação do texto. 